\title{
Erratum to: A genetic approach to the origin of Millepora sp. in the eastern Atlantic
}

\author{
C. López ${ }^{1} \cdot$ S. Clemente ${ }^{1} \cdot$ C. Almeida ${ }^{2} \cdot$ J. C. Hernández $^{1}$. \\ A. Brito ${ }^{1} \cdot$ M. Hernández ${ }^{3}$
}

Published online: 19 May 2015

(C) Springer-Verlag Berlin Heidelberg 2015

\section{Erratum to: Coral Reefs \\ DOI 10.1007/s00338-015-1260-8}

All authors have agreed to add a new author.

New list of authors with additional author is as follows:
C. López, S. Clemente, C. Almeida, J. C. Hernández, A. Brito, M. Hernández.

Instead of:

C. López, S. Clemente, C. Almeida, A. Brito, M. Hernández.

The online version of the original article can be found under doi:10.1007/s00338-015-1260-8.

C. López

kattaissa@gmail.com

1 Departamento de Biología Animal, Edafología y Geología, Facultad de Biología, Universidad de La Laguna (ULL), Avenida Astrofísico Francisco Sánchez, s/n., 38206 La Laguna (Tenerife), Spain

2 Departamento de Engenharias e Ciências do Mar., Universidad de Cabo Verde, Praia, Cape Verde

3 Instituto de Enfermedades Tropicales y Salud Pública de Canarias, Genética. Universidad de La Laguna (ULL), Avenida Astrofísico Francisco Sánchez, s/n., 38206 La Laguna (Tenerife), Spain 